PAPER

\title{
Mirtazapine increases cortical excitability in healthy controls and epilepsy patients with major depression
}

\author{
A Münchau, J M Langosch, W Gerschlager, J C Rothwell, M Orth, M R Trimble
}

J Neurol Neurosurg Psychiatry 2005;76:527-533. doi: 10.1136/jnnp.2004.037010

See end of article for authors' affiliations

.....................

Correspondence to: Dr Alexander Münchau, Department of Neurology, Hamburg University Martinistrasse 52, 20246 Hamburg, Germany; Muenchau@uke. uni-hamburg.de

Received 16 January 2004 In revised form

5 June 2004

Accepted 15 July 2004

\begin{abstract}
Background: Epilepsy is often complicated by depression requiring antidepressant treatment. Such treatment might be proconvulsive.

Objective: To examine the effects of the noradrenergic and specific serotonergic antidepressant mirtazapine on motor cortex excitability in epilepsy patients with depression and in healthy controls, using transcranial magnetic stimulation (TMS).

Methods: Seven clinically depressed epilepsy patients treated with anticonvulsant drugs and six healthy volunteers were studied. Before intake of mirtazapine and 24 hours afterwards (and also three weeks afterwards in the patients), the active and resting motor threshold (AMT, RMT), the size of the motor evoked potential (MEP), the cortical silent period (SP), and intracortical inhibition/facilitation and intracortical facilitatory I wave interactions were determined using single and paired pulse TMS.

Results: At baseline, AMT and RMT were higher $(p=0.049$ and $p=0.04$, respectively) and the ratio SP duration/MEP area greater in patients $(p=0.041)$. In patients but not in healthy subjects AMT was lower 24 hours after intake of mirtazapine $(p=0.028)$. Mirtazapine had no significant effect on the MEP size, duration of the SP, or the ratio of SP duration to MEP size in patients. The duration of the SP was longer $(p=0.037)$ but the ratio of SP duration to MEP size remained similar in healthy subjects after mirtazapine. There were no significant differences in paired pulse measures between the two groups either at baseline or after mirtazapine.

Conclusions: Mirtazapine increased neuronal excitability of pyramidal tract axons in an activated state in both healthy controls and epilepsy patients with major depression.
\end{abstract}

in epilepsy, an increase in cortical excitability or an mbalance between inhibition and excitation of neurones presumably causes seizures. Antiepileptic drugs are known to reduce motor cortex excitability as determined by transcranial magnetic stimulation (TMS), a non-invasive tool to measure the excitability of motor cortical areas. ${ }^{12}$ For instance, antiepileptic drugs that block sodium channel dependent action potentials (for example, phenytoin, carbamazepine, topiramate, or lamotrigine) increase motor thresholds, ${ }^{3-5}$ and GABA-ergic anticonvulsants (such as lorazepam) decrease the tone of intracortical facilitatory neurones. ${ }^{36}$ Epilepsy is often complicated by depression which requires treatment with antidepressant drugs. ${ }^{78}$ Such treatment generally raises concern over provoking seizures or increasing the frequency and duration of seizures, as some antidepressant drugs-particularly tricyclics-are potentially proconvulsive. ${ }^{79}$ This may also include pure noradrenaline reuptake inhibitors (reboxetine, for example, has been found to increase cortical excitability in healthy individuals ${ }^{10}$ ). In contrast, selective serotonin reuptake inhibitors (SSRI) have little or no proconvulsive potential. ${ }^{11}$ For instance, following a single oral dose of sertraline, intracortical facilitation was reduced in healthy subjects. ${ }^{12}$

Given a potential proconvulsive action of noradrenaline reuptake inhibitors on the one hand and the apparent safety of SSRIs on the other, the question arises as to which way a drug with both noradrenergic and specific serotonergic properties-for example, mirtazapine-would affect motor excitability. An advantage of mirtazapine is that it interferes little with the metabolism of other drugs such as anticonvulsants. ${ }^{13}$ As an antagonist at central presynaptic $\alpha 2$ adrenergic autoreceptors, mirtazapine increases the release of noradrenaline. By blocking $\alpha 2$ heteroreceptors on serotonergic neurones it prevents the inhibitory action of noradrenaline on serotonin release. ${ }^{13}$ Although its mode of action is well defined the effects of mirtazapine on motor cortical excitability have not been studied in human subjects. This is important because, as discussed above, antidepressants may increase cortical excitability and, in turn, propagate seizures in patients with epilepsy. In this study we examined the effects of mirtazapine using TMS.

The consequences of altered serotonergic and noradrenergic transmission seem to depend on the state of neuronal networks-for example, the level of cortical excitability. ${ }^{14}$ Thus it is possible that healthy subjects may respond differently to mirtazapine than epilepsy patients treated with anticonvulsant drugs. We therefore compared the effect of mirtazapine on healthy subjects with the effect on depressed epilepsy patients receiving anticonvulsant drug treatment.

To determine effects of mirtazapine on membrane related neuronal excitability we measured resting and active motor thresholds (RMT and AMT, respectively). As a measure of the activity of GABA-ergic transmission we also determined the cortical silent period (SP), intracortical inhibition and facilitation (ICI/ICF), and intracortical facilitatory I wave (indirect wave) interactions using paired pulse paradigms. ${ }^{15} 16$ The silent period reflects inhibitory mechanisms operating both at the spinal and the cortical level. Whereas spinal mechanisms prevail during the first $50 \mathrm{~ms}$ or so, cortical mechanisms predominate later. ${ }^{17-19}$ These later parts reflect

\footnotetext{
Abbreviations: AMT, active motor threshold; BDI, Beck depression inventory; DSM-IV, Diagnostic and Statistical Manual of Mental Disorders, 4th edition; FDI, first dorsal interosseous muscle; ICF, intracortical facilitation; ICl, intracortical inhibition; ISI, interstimulus interval; MEP, motor evoked potential; RMT, resting motor threshold; SP, silent period; SSRI, selective serotonin reuptake inhibitor; TMS, transcranial magnetic stimulation
} 


\begin{tabular}{lllll|}
\hline Table 1 & \multicolumn{4}{l}{ Patient characteristics } \\
\hline Patient & Sex & Age lyears) & Seizure type & Drugs \\
\hline 1 & M & 31 & GTC & Phenobarbitone, topiramate \\
2 & F & 51 & GTC, complex-partial & CBZ, clobazam \\
3 & F & 36 & GTC & PHT, valproate \\
4 & F & 61 & Complex-partial & PHT, clobazam \\
5 & F & 33 & GTC & CBZ, clobazam, ethosuximide \\
6 & F & 45 & GTC, complex-partial & CBZ, clonazepam \\
7 & M & 48 & GTC, juvenile myoclonic & PHT, valproate \\
\hline CBZ, carbamazepine; F, female; GTC, generalised tonic-clonic; M, male; PHT, phenytoin. \\
\hline
\end{tabular}

the effectiveness of long lasting cortical inhibition modulated by GABA-B. ${ }^{20} 21$

Intracortical inhibition at short interstimulus intervals (ISI) $(2-5 \mathrm{~ms})$ is mainly modulated by GABA-A receptors, ${ }^{22}$ whereas intracortical facilitation is controlled by GABA-A and NMDA receptors. ${ }^{23-25}$ The term "I wave" refers to multiple corticospinal volleys following the initial D (direct) wave after electrical motor cortex surface stimulation according to the hypothesis that the $\mathrm{D}$ wave originates from direct and the I wave from indirect (trans-synaptic) activation of corticospinal neurones. ${ }^{26}$ These I waves are suppressed by GABA enhancing drugs. ${ }^{27}$

\section{METHODS}

\section{Subjects and patients}

Six healthy right handed volunteers served as controls (four men, two women; mean age 39 years, range 36 to 58), while seven patients with primary epilepsy and major depression (two men, five women; mean age 43.6 years, range 31 to 61 ) were recruited from the epilepsy clinic at the National Hospital for Neurology and Neurosurgery, Queen Square, London, as the index cases. There were no sex or age differences between the patients and the healthy subjects. All epilepsy patients were taking at least one antiepileptic drug. The characteristics of the patients are given in table 1. All participants gave their informed written consent before participation. The local ethics committee approved the study protocol.

\section{Study design}

At study entry patients had a full psychiatric evaluation including a structured clinical interview for a DSM-IV diagnosis of depression ${ }^{28}$ and they completed the Beck depression inventory (BDI). ${ }^{29}$ All patients met the DSM-IV criteria for current major depression and had a BDI score of more than 18.

Mirtazapine is absorbed rapidly and well after oral administration, reaching peak plasma concentrations within about two hours. The mean half life is approximately 20 to 40 hours, with a pharmacologically antidepressive active metabolite, justifying once a day dosing. ${ }^{30}$ Thus all subjects received a single $30 \mathrm{mg}$ dose of mirtazapine after baseline TMS measurements and were studied again 24 hours later. Patients but not healthy subjects continued to take $30 \mathrm{mg}$ of mirtazapine once daily and were investigated again three weeks later. At that time, patients completed the BDI again, and as a measure of compliance plasma concentrations of mirtazapine were determined in the patients at the Organon Laboratories in Cologne (Germany).

For the duration of the study anticonvulsant drug treatment remained unchanged. Patients or their carers documented the occurrence of seizures for three weeks before and three weeks after the first dose of mirtazapine using a seizure diary, and recorded any adverse events of TMS or treatment with mirtazapine.

\section{Transcranial magnetic stimulation}

For TMS measurements subjects were seated in a comfortable armchair. The right arm was supported by a pillow to allow complete relaxation. Subjects were instructed to relax but to keep their eyes open and fixate a visual target directly in front of them. Surface electromyograms (EMG) were recorded with silver/silver chloride disc electrodes placed in a differential pair over the right first dorsal interosseous (FDI) muscle, using a belly-tendon montage. The earth (ground) was placed at the wrist. The EMG signals were amplified, analogue filtered $(32 \mathrm{~Hz}$ to $1 \mathrm{kHz}$; Toennies amplifier), and acquired at a sampling rate of $5 \mathrm{kHz}$. Data were stored on a personal computer for off-line analysis (Signal Software, Cambridge Electronic Design (CED), Cambridge, UK). EMG activity was monitored continuously during the experiments using an oscilloscope and loudspeakers for visual and auditory feedback. Trials where the target muscle was not relaxed were excluded from the analysis because voluntary contraction of the target muscle has profound effects on intracortical excitability. ${ }^{31}$ Peak to peak amplitude of motor evoked potentials (MEP), the area under the curve of the MEP, and the silent period duration were measured with in-house software.

Magnetic stimuli were given with a hand held figure of eight shaped coil with an outer winding diameter of $70 \mathrm{~mm}$ (Magstim Co, Whitland, Dyfed, UK) connected through a bistim module to two high power Magstim 200 magnetic stimulators (Magstim Co). The magnetic stimulus had a nearly monophasic pulse configuration with a rise time of about $100 \mu \mathrm{s}$, decaying back to zero over about $0.8 \mathrm{~ms}$. The coil was placed tangentially to the scalp with the handle pointing backwards and laterally at a $45^{\circ}$ angle away from the midline, approximately perpendicular to the line of the central sulcus, inducing a posterior-anterior electrical current flow in the brain. This orientation was chosen on the basis that the lowest motor threshold is achieved when the induced electrical current in the brain flows approximately perpendicular to the line of the central sulcus. ${ }^{32}{ }^{33}$ The coil was held by hand in relation to marks made on the scalp (see below).

The site where stimuli of slightly suprathreshold intensity consistently produced the largest motor evoked potentials (MEPs) with the steepest negative slope in right FDI muscle (referred to as the "motor hot spot") was marked with a red wax pen by drawing a semilunar line following the anterior bifurcation of the coil and a straight line indicating the orientation of the coil handle.

\section{Motor thresholds}

As a measure of membrane related neuronal excitability we determined RMT and AMT, expressed as a percentage of maximum stimulator output. RMT was defined as the stimulus intensity that produces an MEP of more than 50 $\mu \mathrm{V}$ in five of 10 consecutive trials in the relaxed FDI. AMT was defined as the lowest stimulus intensity at which MEPs 


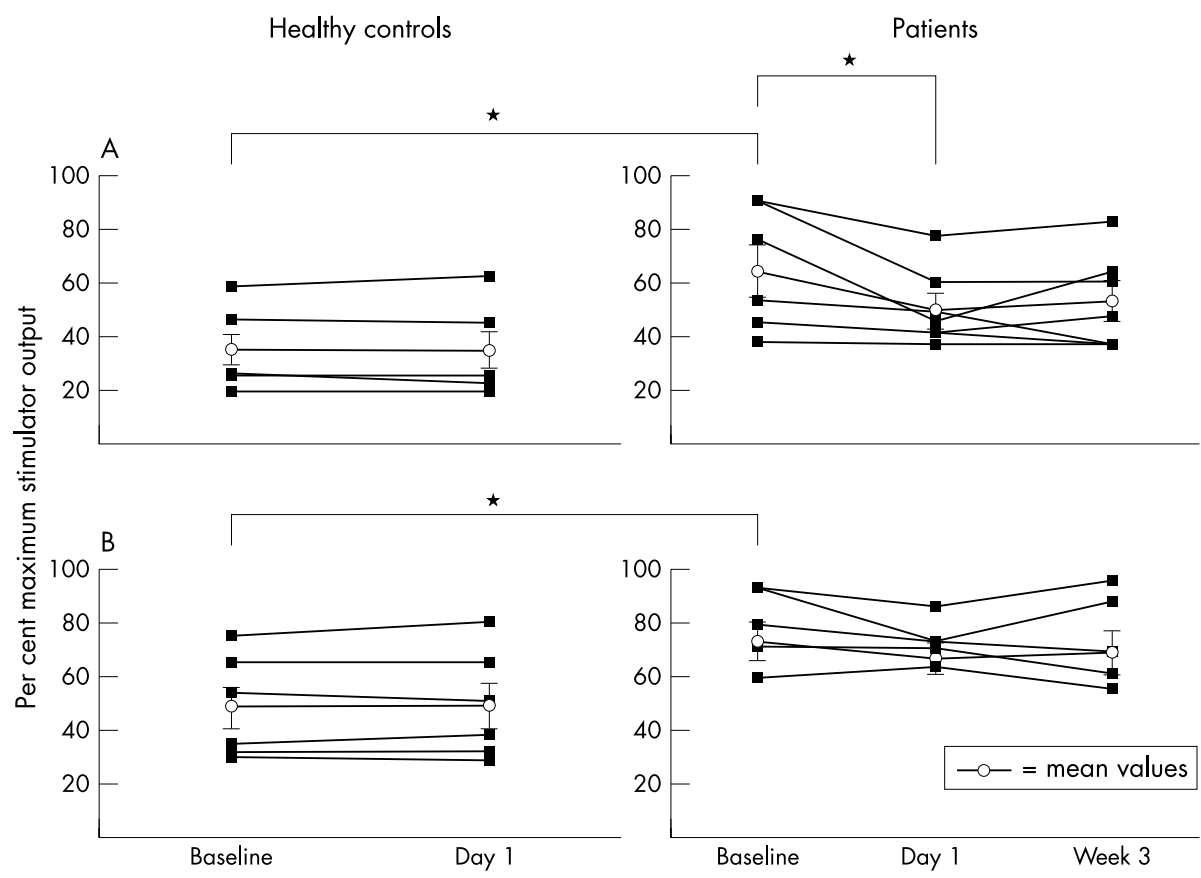

Figure 1 Motor thresholds (per cent of maximum stimulator output). (A) Active motor thresholds. (B) Resting motor thresholds. Black squares represent individual data, white circles are mean values. Error bars indicate SEM. ${ }^{*} \mathrm{p}<0.05$.

of $150 \mu \mathrm{V}$ amplitude were elicited in the tonically contracting FDI muscle in five of 10 consecutive trials.

\section{Cortical silent period}

The duration of the cortical silent period was determined during approximately $50 \%$ of maximum isometric voluntary contraction of the right FDI muscle, which was monitored using acoustic feedback through a loudspeaker and visually on an oscilloscope. Ten single suprathreshold TMS pulses with an intensity of $150 \%$ of AMT were applied. The SP was measured from the onset of the MEP to the resumption of continuous EMG activity. The size of the corresponding MEP (that is, the area under the curve) was measured, and a ratio of SP duration to MEP area calculated for each individual trial as previously described. ${ }^{34}$

\section{Intracortical inhibition and facilitation}

ICI and ICF were evaluated using paired magnetic pulses as described by Kujirai et al. ${ }^{15}$ The second (test) stimulus was set at an intensity that, when it was given alone, evoked an EMG response of approximately 0.5 to $1.0 \mathrm{mV}$ peak to peak amplitude. As we were interested in changes in intracortical excitability we set the intensity of the first (conditioning) stimulus to a relatively low value of $80 \%$ AMT to avoid floor or ceiling effects. We used the following nine different interstimulus intervals (ISI): 2, 3, 4, 5, 6, 7, 10, 15, and $20 \mathrm{~ms}$. These 10 conditions (test pulse given alone and nine conditioned pulses at different ISIs) were applied in three blocks of 50 trials with an interval of five seconds between trials. In each block the control condition (test pulse given alone) was tested 20 times and three of the conditioning test stimuli 10 times. The order of the conditions was randomised. The mean peak to peak amplitude of the conditioned MEP, at each ISI, was expressed as a percentage of the mean peak to peak amplitude of the unconditioned test pulse. In our statistical analysis (see below) we averaged adjacent time points over the period of inhibition at 2, 3, and $4 \mathrm{~ms}$ ISI, over the period of facilitation at 10, 15, and $20 \mathrm{~ms}$ ISI, and for the intermediate ISIs of 5,6 , and $7 \mathrm{~ms}$ to increase the power of our analysis. Thus the factor ISI had three levels.

\section{Intracortical facilitatory I wave interaction}

I waves were studied as described by Ziemann et al, ${ }^{16} 27$ using a slightly modified protocol where the intensity of the first stimulus (S1) was set at $110 \%$ RMT and that of the second stimulus (S2) at 105\% RMT. Previous studies have shown that the conditioned MEPs elicited by S1 plus S2 are larger than those produced by S1 alone at ISIs of around 1.5, 2.7, and $4.2 \mathrm{~ms}$. Here we focused on the first and second of these peaks and thus tested ISIs from 1.0 to $3.4 \mathrm{~ms}$ in $0.2 \mathrm{~ms}$ steps ( = 12 ISIs). The 14 conditions ( $\mathrm{S} 1$ and S2 given alone and 12 conditioned pulses at different ISIs) were applied in four blocks of 50 trials with an interval of five seconds between trials. In each block S1, S2, and three conditioned pulses were tested 10 times. The order of the conditions was randomised. The mean peak to peak amplitude of the conditioned MEP, at each ISI, was expressed as a percentage of the mean peak to peak amplitude of the sum of S1 and S2. The three adjacent ISIs resulting in the maximum sum of MEP values were identified for each of the two MEP peaks and averaged.

\begin{tabular}{llll} 
Table 2 & Silent period measurements \\
\hline Variable & & \multicolumn{1}{l}{ Controls } & Patients \\
\hline Duration $(\mathrm{ms})$ & Baseline & $96.4(42.5)$ & $145.4(54.8)$ \\
& Day 1 & $122.4(33.9)^{*}$ & $162.4(49.2)$ \\
MEP area $(\mu \vee \mathrm{ms})$ & Week 3 & - & $154.8(53)$ \\
& Baseline & $467.9(243.5)$ & $521.3(175.5)$ \\
& Day 1 & $550.3(207.8)$ & $609.4(132.0)$ \\
Ratio duration/MEP area & Week 3 & - & $686.3(237.8)$ \\
& Baseline & $0.22(0.023)$ & $0.28(0.056) \dagger$ \\
& Day 1 & $0.24(0.06)$ & $0.27(0.063)$ \\
& Week 3 & - & $0.25(0.062)$ \\
\hline
\end{tabular}

${ }^{*} p=0.037$ (comparison with baseline); $\uparrow p=0.041$ (comparison between groups).

MEP, motor evoked potential. 


\section{Statistical analyses}

Subjects' characteristics (sex and age) and baseline measurements between healthy subjects and patients were compared using $\mathrm{X}^{2}$ and Student's $t$ tests. In healthy subjects baseline and post-mirtazapine AMT, RMT, MEP amplitudes at rest (unconditioned control MEPs during measurements of ICI and ICF), the area of the MEP preceding the SP, the duration of the SP, and the ratio of SP duration to MEP area were compared using paired samples $t$ tests. In patients we compared measurements at baseline, on day 1 , and at three weeks using a one way repeated measures analysis of variance (ANOVA) with time as within-subjects factor. For post hoc comparisons between baseline measurements and those after intake of mirtazapine on day 1 and at three weeks paired samples $t$ tests were used. Next, to examine whether the effects of mirtazapine on AMT, MEP area preceding the SP, and the duration of the SP differed between controls and patients, two factor repeated measures ANOVAs with the factors subjects and time (two levels: before and one day after intake of mirtazapine) were carried out. Two factor repeated measures ANOVAs with the factors subject and ISI (three levels) or peak (two levels) were done to compare baseline ICI/ICF and I wave curves between groups. Additional two factor repeated measures ANOVAs with the factors time (three levels in patients and two levels in healthy subjects) and ISI (three levels) or peak (two levels) examined the effects of mirtazapine on ICI/ICF and I wave interaction, respectively.

Mauchley's test examined sphericity of the data. The Greenhouse-Geisser correction was used to correct for nonsphericity. Conditional on a significant $\mathrm{F}$ value, post-hoc paired samples $t$ tests were carried out.

To test whether mirtazapine had an antidepressant effect in patients we compared BDI scores at baseline with scores at three weeks using the Wilcoxon signed rank test. To determine whether there was a correlation between changes in AMT and changes in BDI score we used Spearman's rank correlation coefficient.

For all statistical analyses a probability (p) value of $<0.05$ was considered significant.

\section{RESULTS}

\section{Clinical effects, adverse effects, and compliance}

None of the subjects reported adverse effects after TMS. According to the patients' seizure diaries there was no increase in seizure frequency, duration, or severity during the study period. All patients and five of the six healthy subjects reported mild to moderate sedation, mild dizziness, and a dry mouth, starting a few hours after the mirtazapine dose and lasting for up to 48 hours. Sedation resolved within another five to 10 days in most patients. No other side effects were reported during the study period. Clinical assessment did not reveal a major antidepressant effect of mirtazapine and there was no major change in the mean (SD) BDI score: 56.8 (5.9) at baseline and $55.4(4.1)$ at three weeks; $z=-0.85, \mathrm{p}=0.39$; Wilcoxon signed rank test.

Further follow up (for 25 (15.4) weeks) showed that four of the seven patients stopped mirtazapine after a mean of 7.5 (4.7) weeks because of lack of efficacy $(n=3)$, persisting unacceptable sedation $(n=1)$, or weight gain $(n=1)$. None of these four patients reported an increase in seizure frequency during that time. The remaining three patients remained on mirtazapine for a further 36.7 (13.6) weeks. In these patients there was a mild improvement in depression on clinical grounds but no change in seizure frequency during the time of follow up.

Mirtazapine was detected in the blood of all patients at three weeks. Thus all patients were considered compliant. Mirtazapine blood levels varied considerably ( 1.89 to $34.0 \mathrm{ng}$ / ml; mean (SEM) value $13.09(5.04) \mathrm{ng} / \mathrm{ml})$. This can be explained by the variable interval between drug intake and the times when the blood samples were taken (between 10 and 24 hours), as plasma concentrations of mirtazapine are inversely related to the time after the last dose (for discussion see Timmer et $^{a l^{35}}$ ).

\section{Motor thresholds and MEP size}

At baseline both AMT and RMT were significantly higher in the epilepsy patients than in the healthy controls $(\mathrm{T}(11)=2.2, \mathrm{p}=0.049$, and $\mathrm{T}(11)=2.3, \mathrm{p}=0.04$, respectively; Student's $t$ test; fig 1). In healthy subjects there was no change in AMT (34.8 (14.7) at baseline and 34.7 (16.5) on day 1) or RMT (48.5 (19.0) at baseline and 49.2 (20.2) on day 1) after intake of mirtazapine (paired samples $t$ test; fig 1). In patients, however, mirtazapine led to a significant reduction in AMT (from 60.1 (25.2) at baseline to 47.7 (16.8) on day $\mathrm{l}$ and 50.4 (19.4) at three weeks; $\mathrm{F}(2,12)=4.8$; $\mathrm{p}=0.028$; repeated measures ANOVA), but not RMT (72.4 (18.0) at baseline, 66.7 (15.0) on day l, and 67.3 (20.4) at three weeks; fig 1). Post-hoc paired samples $t$ tests (comparisons of baseline AMT with AMT on day 1 and at three weeks) showed that AMT was significantly lower on day 1 compared with baseline measurements $(\mathrm{T}(6)=2.5$; $\mathrm{p}=0.044)$. AMTs were still lower at three weeks, but this was no longer significant. Despite the fall in AMT in patients associated with mirtazapine, on day 1 AMT and RMT were still higher in epilepsy patients than in the healthy subjects, but this difference was no longer significant.

We went on to test the effect of mirtazapine on thresholds in a repeated measures ANOVA model that included both healthy controls and patients at baseline and on day 1 . The results confirm that mirtazapine was associated with a fall in AMT in patients but not controls (interaction of subjects $\times$ time; $\mathrm{F}(1,11)=5.2 ; \mathrm{p}=0.04)$. There was no correlation between AMT and BDI scores at baseline or between changes of AMT and changes of BDI scores.

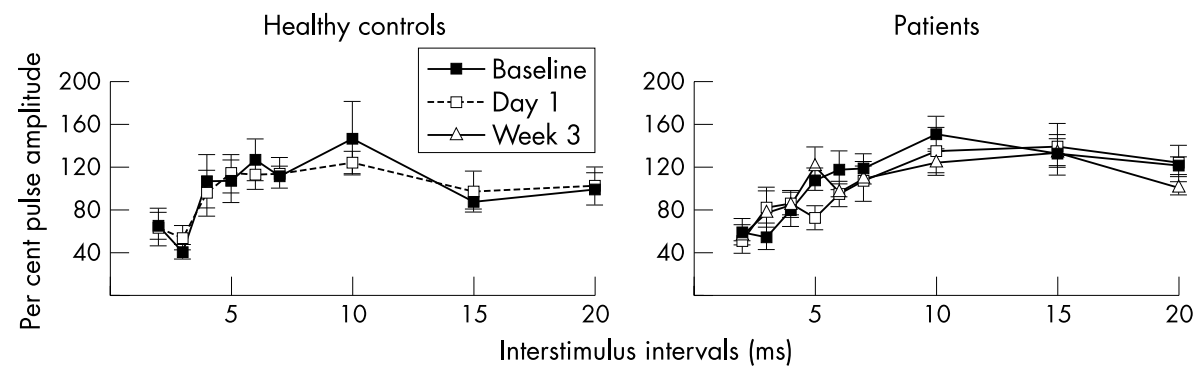

Figure 2 Intracortical inhibition and facilitation. On the $x$ axis the different interstimulus intervals are shown. On the $y$ axis the mean amplitude of the conditioned MEP is shown as a percentage of the mean test pulse amplitude. Baseline paired pulse curves and those after intake of mirtazapine are superimposed. Error bars indicate SEM. 

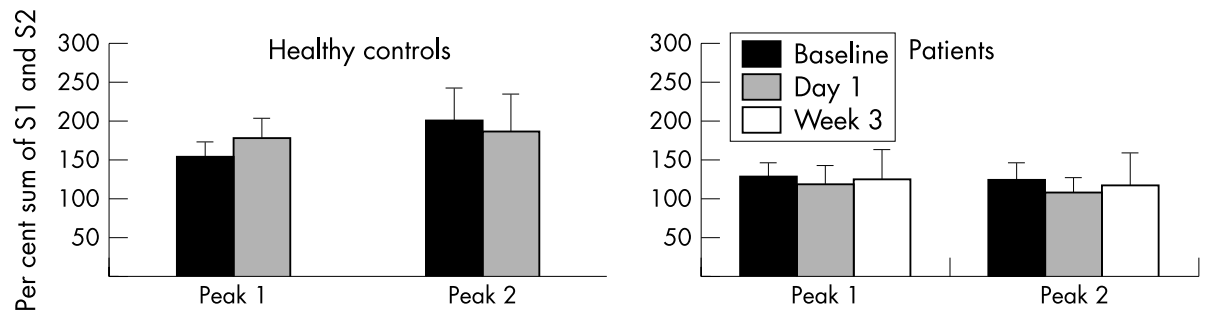

Figure 3 Facilitatory I wave peaks. On the $y$ axis the mean amplitude of the conditioned MEP is shown as a percentage of the mean sum of S1 and S2. Peak 1 is the mean MEP amplitudes at ISls of 1.4, 1.6, and $1.8 \mathrm{~ms}$, and peak 2 that at ISIs of 2.8, 3.0, and 3.2. Error bars indicate SEM.

Mean baseline MEP size at rest was smaller, though nonsignificantly so, in patients than in the healthy subjects, at $0.63(0.6) v 0.96(0.6) \mathrm{mV}$. There was no significant effect of mirtazapine on mean MEP amplitudes at rest in either group.

\section{Cortical silent period}

At baseline, the average duration of the SP was longer, although not significantly so, in epilepsy patients (table 2 ). There was no baseline difference of MEP area between groups. However, the ratio of SP duration to MEP area was significantly greater in epilepsy patients $(\mathrm{T}(10)=2.4$; $\mathrm{p}=0.041$, Student's $t$ test). In healthy subjects the duration of the SP after mirtazapine was significantly longer than at baseline $(\mathrm{T}(4)=-3 ; \mathrm{p}=0.037)$. MEP area also increased, with a trend on statistical testing $(\mathrm{T}(4)=-2.3 ; \mathrm{p}=0.076)$. The ratio of SP duration/MEP area remained similar (table 2 ). Treatment with mirtazapine had no significant effect on the duration of the SP in patients. The MEP area increased, but the increase was not significant. The ratio of SP duration to MEP area remained unchanged (table 2). Two factor repeated measures ANOVA confirmed that there were no differential effects of mirtazapine on these variables in the two groups (no interaction of time $\times$ subjects).

\section{Paired pulse TMS measurements}

Baseline ICI/ICF curves of patients and healthy subjects were similar and they remained similar over time (two factor repeated measures ANOVA with the factors subject and ISI; fig 2).

The effects of mirtazapine on I wave interaction are shown in fig 3. As outlined in Methods we averaged the three adjacent ISIs, resulting in the maximum sum of MEP values. The comparison of the conditioned MEP amplitudes at ISIs of 1.4, 1.6, and $1.8 \mathrm{~ms}$ (peak 1) and 2.8, 3.0, and $3.2 \mathrm{~ms}$ (peak 2) showed no significant difference between the two groups at baseline. Mirtazapine had no effect on I wave interaction in either group.

Plasma concentrations of mirtazapine were not correlated with TMS measurements of cortical excitability because the correlation between plasma mirtazapine and the CNS effects of mirtazapine is unclear.

\section{DISCUSSION}

This study confirms previous reports showing that epilepsy patients on anticonvulsant drugs have higher active and resting motor thresholds than controls. ${ }^{36}{ }^{37}$ Moreover, the ratio of silent period duration to MEP area was significantly larger in patients, suggesting that the inhibitory mechanisms underlying the silent period are activated by a TMS pulse out of proportion to the size of the preceding MEP. This means that the motor cortex of these patients was less excitable than the motor cortex of controls.

We went on to examine the neurophysiological effects of mirtazapine on motor cortical excitability in these patients and in the healthy controls. In the healthy controls mirtazapine led to an increase in the duration of the cortical silent period. This could suggest that in the healthy motor cortex mirtazapine enhances inhibition within the inhibitory circuits that underlie the cortical silent period. However, the MEP size preceding the silent period (that is, the MEP area under preactivation elicited with a TMS pulse of $150 \%$ AMT) increased relative to the silent period. MEP size and silent period duration are strongly correlated. ${ }^{34}$ As the pyramidal tract neurones may give off recurrent inhibitory collaterals, ${ }^{38}$ this suggests that the neural elements responsible for the MEP size under preactivation and silent period duration are linked. We therefore think that the increase in silent period duration in healthy subjects is a consequence of the increase in MEP size. In fact, the ratio of silent period duration to MEP area was unchanged. Taken together, the data in healthy control subjects indicate that mirtazapine increases the excitability of axons of pyramidal tract neurones during voluntary contraction.

The duration of the silent period has been found to be unchanged by sertraline, a selective 5-HT reuptake inhibitor, ${ }^{12}$ or zolmitriptan, a 5 -HT $1 \mathrm{~B} / \mathrm{lD}$ receptor agonist, ${ }^{39}$ in healthy subjects. However, the relation of MEP size and silent period duration was not determined in those studies, so a direct comparison is not possible. Effects of noradrenaline enhancing drugs on the silent period have not been studied previously.

In patients, mirtazapine treatment was associated with a fall in AMT and, as in the controls, a non-significant increase in the MEP size preceding the silent period, but it did not change silent period duration or the ratio of silent period duration to MEP area. This suggests that in both healthy controls and epilepsy patients with major depression, mirtazapine increases neuronal excitability of pyramidal tract axons in an activated state. This could reflect either the noradrenaline enhancing properties of the drug or the serotonin enhancing properties, or a combination of both. A noradrenergic effect would be in accordance with a previous TMS study where a single dose of the antidepressant reboxetine, a selective noradrenaline reuptake inhibitor, lowered RMT in a group of healthy subjects. ${ }^{10}$ However, as threshold values were not provided in that study, a direct comparison with our study is not possible. In another study, reboxetine did not alter AMT in a group of healthy subjects whose AMT were very similar to those in our control group $(35.8 \%$ stimulator output $v 34.8 \%){ }^{40}$ However, reboxetine and yohimbine, a presynaptic $\alpha 2$ antagonist, produced an increase in ICF in healthy subjects. ${ }^{10} 4041$ This confirms that noradrenaline can have excitability enhancing effects in the human motor cortex. On the other hand, it has been shown that the 5-HT reuptake inhibitor sertraline diminishes ICF. ${ }^{12}$ Apparently, serotonin and noradrenaline have opposite actions on facilitatory interneurones in the human motor cortex. As mirtazapine has both noradrenaline and 5-HT enhancing actions, these effects may balance each other. This could be the reason why ICF was not altered after mirtazapine in our study. 
ICI and ICF were also not significantly different between groups at baseline. At first sight this may appear surprising, as previous TMS studies have shown that the GABA-ergic anticonvulsants-for example, lorazepam, gabapentin, and vigabatrin - that some of our patients were taking can lead to a decrease in ICF. $^{36}$ However, there are methodological differences between previous studies and the present experiments that have to be taken into account. We used a conditioning pulse with a relatively low intensity of $80 \%$ AMT, whereas in the studies cited above the conditioning pulse had a strength of $95 \%$ AMT. ${ }^{36}$ As conditioning stimuli with an intensity of $80 \%$ AMT produce similar intracortical inhibition but less intracortical facilitation of the test pulse than those with an intensity of $95 \% \mathrm{AMT}^{15}$ the lack of a reduction in ICF in our epilepsy patients might be explained by a floor effect. There was no significant group difference of the facilitatory interaction of I waves at baseline. I wave peaks were unaffected by mirtazapine.

The differential effect in controls and patients (a fall of AMT in patients, an increase in silent period duration in controls) may reflect floor (AMT in controls) or ceiling effects (maximum silent period duration in patients). For instance, the duration of the silent period can be increased by GABA-B receptor activation. ${ }^{2021}$ The GABA-B receptor dependent inhibitory postsynaptic potential peaks at 150 to $200 \mathrm{~ms}^{42}$ This could explain why increases in the silent period duration were only present in healthy subjects in whom baseline duration was about $100 \mathrm{~ms}$ but not in epilepsy patients where the duration at baseline was in the range of $150 \mathrm{~ms}$.

A pharmacological interaction of mirtazapine with anticonvulsants is less likely as an explanation for the effects we observed in our epilepsy patients. Mirtazapine is degraded in human liver microsomes, where cytochrome P450 is involved in the formation of 8-hydroxy metabolites. It is a weak competitive inhibitor of several cytochome P450 isoenzymes (CYP 1A2, CYP 2C9/19, CYP 2D6, and CYP 3A4) and a substrate of CYP $1 \mathrm{~A} 2$ and CYP $3 \mathrm{~A}$, but is not considered to play a significant role at cytochrome $\mathrm{p} 450$ level with respect to the metabolism of coadministered drugs. ${ }^{43}$ Had there been an interference with anticonvulsants this would most probably have resulted in an increase in plasma concentrations of anticonvulsants because of interference with their metabolism. This would presumably have further increased rather than decreased the motor thresholds.

Alternatively, premedication with sodium channel blocking anticonvulsants might have interfered with the physiological mode of action of mirtazapine. Conceivably, such premedication could have made patients susceptible to threshold effects.

Finally, one could speculate that motor threshold effects of noradrenaline and 5-HT only occur physiologically when baseline motor thresholds are increased, regardless of the underlying cause.

Taken together our data suggest that mirtazapine enhances cortical excitability in healthy controls and in epilepsy patients with depression. Our results could have clinical implications, as the increase in cortical excitability in patients with epilepsy and major depression may be associated with an increase in seizures. For the duration of our study and a short follow up period thereafter our patients did not report major changes in seizure frequency. However, both the small number of patients and the short duration of the trial limit the power of our study in this respect. Longer studies with a larger number of patients should address this question.

There was no major antidepressant effect of mirtazapine in this group of epilepsy patients during the study period, and even during a short follow up beyond. However, it was not the objective of our study to assess the antidepressant effect of mirtazapine or to correlate the electrophysiological changes with a clinical effect on depression. As the relation between motor thresholds and mood changes is controversial, ${ }^{44-46}$ it would be interesting to examine whether the reduction in AMT in patients with epilepsy and major depression on mirtazapine is sustained over longer periods, and whether this correlates with an antidepressant effect.

\section{Authors' affiliations}

A Münchau, J M Langosch, W Gerschlager, J C Rothwell, M Orth, Sobell Department of Motor Neuroscience and Movement Disorders, Department of Clinical and Experimental Epilepsy, Institute of Neurology, National Hospital for Neurology and Neurosurgery, Queen Square, London, UK

M R Trimble, Raymond Way Unit, Department of Clinical and Experimental Epilepsy, Institute of Neurology, National Hospital for Neurology and Neurosurgery

Competing interests: none declared

\section{REFERENCES}

1 Rothwell JC. Physiological studies of electric and magnetic stimulation of the human brain. Electroencephalogr Clin Neurophysiol Suppl 1991;43:29-35.

2 Civardi C, Cantello R, Asselman P, et al. Transcranial magnetic stimulation can be used to test connections to primary motor areas from frontal and medial cortex in humans. Neuroimage 2001; 14:1444-53.

3 Ziemann U, Lonnecker S, Steinhoff BJ, et al. Effects of antiepileptic drugs on motor cortex excitability in humans: a transcranial magnetic stimulation study. Ann Neurol 1996:40:367-78.

4 Manganotti $P$, Bongiovanni LG, Zanette $G$, et al. Cortical excitability in patients after loading doses of lamotrigine: a study with magnetic brain stimulation. Epilepsia 1999;40:316-21.

5 Chen R, Samii A, Canos M, et al. Effects of phenytoin on cortical excitability in humans. Neurology 1997;49:881-3.

6 Ziemann U, Lonnecker S, Steinhoff BJ, et al. The effect of lorazepam on the motor cortical excitability in man. Exp Brain Res 1996;109:127-35.

7 Trimble M. Non-monoamine oxidase inhibitor antidepressants and epilepsy: a review. Epilepsia 1978;19:241-50.

8 Mendez MF, Doss RC, Taylor JL, et al. Depression in epilepsy. Relationship to seizures and anticonvulsant therapy. J Nerv Ment Dis 1993;181:444-7.

9 Trimble MR. New antidepressant drugs and the seizure threshold. Neuropharmacology 1980;19:1227-8.

10 Herwig U, Braver K, Connemann B, et al. Intracortical excitability is modulated by a norepinephrine-reuptake inhibitor as measured with pairedpulse transcranial magnetic stimulation. Psychopharmacology 2002;164:228-32.

11 Cooper GL. The safety of fluoxetine - an update. Br J Psychiatry Suppl 1988:77-86.

12 Ilic TV, Korchounov A, Ziemann U. Complex modulation of human motor cortex excitability by the specific serotonin re-uptake inhibitor sertraline. Neurosci Lett 2002;319:116-20.

13 de Boer T. The pharmacologic profile of mirtazapine. J Clin Psychiatry 1996:57(suppl 4):19-25.

14 Andrade R. Regulation of membrane excitability in the central nervous system by serotonin receptor subtypes. Ann NY Acad Sci 1998;861:190-203.

15 Kujirai T, Caramia MD, Rothwell JC, et al. Corticocortical inhibition in human motor cortex. J Physiol (Lond) 1993;471:501-19.

16 Ziemann U, Tergau F, Wassermann EM, et al. Demonstration of facilitatory I wave interaction in the human motor cortex by paired transcranial magnetic stimulation. J Physiol (Lond) 1998:511:181-90.

17 Fuhr P, Agostino R, Hallett M. Spinal motor neuron excitability during the silent period after cortical stimulation. Electroencephalogr Clin Neurophysiol 1991:81:257-62.

18 Inghilleri M, Berardelli A, Cruccu G, et al. Silent period evoked by transcranial stimulation of the human cortex and cervicomedullary junction. J Physiol (Lond) 1993;466:521-34.

19 Roick H, von Giesen HJ, Benecke R. On the origin of the postexcitatory inhibition seen after transcranial magnetic brain stimulation in awake human subjects. Exp Brain Res 1993;94:489-98.

20 Werhahn KJ, Kunesch E, Noachtar S, et al. Differential effects on motorcortical inhibition induced by blockade of GABA uptake in humans. J Physiol (Lond) 1999;517:591-7.

21 Siebner HR, Dressnandt J, Auer C, et al. Continuous intrathecal baclofen infusions induced a marked increase of the transcranially evoked silent period in a patient with generalized dystonia. Muscle Nerve 1998;21:1209-12.

22 Reis J, Tergau F, Hamer HM, et al. Topiramate selectively decreases intracortical excitability in human motor cortex. Epilepsia 2002;43:1149-56.

23 Schwenkreis P, Liepert J, Witscher K, et al. Riluzole suppresses motor cortex facilitation in correlation to its plasma level. A study using transcranial magnetic stimulation. Exp Brain Res 2000;135:293-9.

24 Ziemann U, Chen R, Cohen LG, et al. Dextromethorphan decreases the excitability of the human motor cortex. Neurology 1998;51:1320-4. 
25 Liepert J, Schwenkreis $\mathrm{P}$, Tegenthoff $M$, et al. The glutamate antagonist riluzole suppresses intracortical facilitation. J Neural Transm 1997; 104:1207-14

26 Patton HD, Amassian VE. Single and multiple-unit analysis of cortical stage of pyramidal tract activation. J Neurophysio 1954;17:345-63.

27 Ziemann U, Tergau F, Wischer S, et al. Pharmacological control of facilitatory I-wave interaction in the human motor cortex. A paired transcranial magnetic stimulation study. Electroencephalogr Clin Neurophysiol 1998;109:321-30.

28 First MB, Spitzer RL, William JBW, et al. Structured clinical interview for DSMIV (SCID). Washington: American Psychiatry Press, 1995.

29 Beck AT, Ward CH, Mendelson M, et al. An inventory for measuring depression. Arch Gen Psychiatry 1961;4:561-71.

30 Timmer CJ, Paanakker JE, Vrijmoed-de Vries M. Mirtazapine pharmacokinetics with two dosage regimens and two pharmaceutical formulations. Pharmacol Res 1997;14:98-102.

31 Ridding MC, Taylor JL, Rothwell JC. The effect of voluntary contraction on cortico-cortical inhibition in human motor cortex. J Physiol (Lond) 1995;487:541-8

32 Brasil-Neto JP, Cohen LG, Panizza M, et al. Optimal focal transcranial magnetic activation of the human motor cortex: effects of coil orientation, shape of the induced current pulse, and stimulus intensity. J Clin Neurophysiol 1992;9:132-6.

33 Mills KR, Boniface SJ, Schubert M. Magnetic brain stimulation with a double coil: the importance of coil orientation. Electroencephalogr Clin Neurophysiol 1992;85:17-21.

34 Orth M, Rothwell JC. The cortical silent period: intrinsic variability and relation to the waveform of the transcranial magnetic stimulation pulse. Clin Neurophysiol 2004;115:1076-82.

35 Timmer CJ, Lohmann AAM, Mink LPA. Pharmakokinetic dose-proportionality study at steady state of Mirtazapine from Remeron tablets. Hum Psychopharmacol 1995;10:97-106.
36 Reutens DC, Berkovic SF, Macdonell RA, et al. Magnetic stimulation of the brain in generalized epilepsy: reversal of cortical hyperexcitability by anticonvulsants. Ann Neurol 1993;34:351-5.

37 Michelucci R, Passarelli D, Riguzzi $P$, et al. Transcranial magnetic stimulation in partial epilepsy: drug-induced changes of motor excitability. Acta Neurol Scand 1996;94:24-30.

38 Phillips CG, Porter R. Corticospinal neurones. Their role in movement. London: Academic Press, 1977.

39 Werhahn KJ, Forderreuther S, Straube A. Effects of the serotonin 1B/1D receptor agonist zolmitriptan on motor cortical excitability in humans. Neurology 1998;51:896-8.

40 Plewnia C, Hoppe J, Hiemke C et al. Enhancement of human corticomotoneuronal excitability by the selective norepinephrine reuptake inhibitor reboxetine. Neurosci Lett 2002;330:231.

41 Plewnia C, Bartels M, Cohen L, et al. Noradrenergic modulation of human cortex excitability by the presynaptic alpha(2)-antagonist yohimbine. Neurosci Lett 2001;307:41-4.

42 Mott DD, Lewis DV. The pharmacology and function of central GABAB receptors. Int Rev Neurobiol 1994;36:97-223.

43 Dahl M-L, Voortman G, Alm C, et al. In vitro and in vivo studies on the disposition of mirtazapine in humans. Clin Drug Invest 1997;13:37-46.

44 Maeda F, Keenan JP, Pascual-Leone A. Interhemispheric asymmetry of motor cortical excitability in major depression as measured by transcranial magnetic stimulation. Br J Psychiatry 2000;177:169-73.

45 Triggs WJ, McCoy KJ, Greer R, et al. Effects of left frontal transcranial magnetic stimulation on depressed mood, cognition, and corticomotor threshold. Biol Psychiatry 1999;45:1440-6.

46 Dolberg OT, Dannon PN, Schreiber S, et al. Magnetic motor threshold and response to TMS in major depressive disorder. Acta Psychiatr Scand 2002; 106:220-3 